\title{
Effect of Clinical and Histological Chorioamnionitis on the Outcome of Preterm Infants
}

\author{
Nehad Nasef, MD ${ }^{1}$ Abd Elazeez Shabaan, MD ${ }^{1}$ Patti Schurr, RN (EC), MSc ${ }^{1}$ \\ Dolores laboni, BScPhm ${ }^{1}$ Julie Choudhury, Pharm.D. ${ }^{1}$ Paige Church, MD, FAAP ${ }^{1,2}$ \\ Michael S. Dunn, MD, FRCPC ${ }^{1,2}$
}

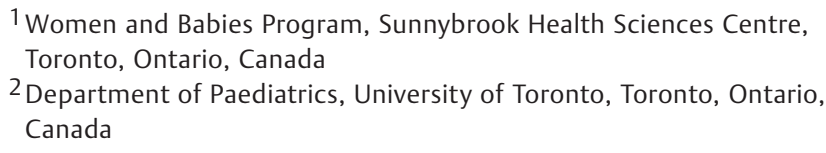
Am J Perinatol 2013;30:59-68.
Address for correspondence and reprint requests Michael S. Dunn, MD, FRCPC, Department of Newborn and Developmental Paediatrics, Sunnybrook Health Sciences Centre, Room M4-222, 2075 Bayview Avenue, Toronto, ON, Canada M4N 3M5 (e-mail: michael.dunn@sunnybrook.ca).

\begin{abstract}
Keywords

- chorioamnionitis

- preterm infants

- perinatology

- neurodevelopment

Chorioamnionitis contributes to neonatal and maternal morbidity and mortality. We aimed to evaluate of the impact of clinical and histological chorioamnionitis on mortality and morbidity of preterm infants. Maternal and neonatal data were collected in a retrospective cohort of preterm infants less than 30 weeks' gestation. Infants were divided into three groups: those born to mothers with clinical chorioamnionitis, histological chorioamnionitis, or no chorioamnionitis. Of 274 identified preterm infants, 33 infants were born to mothers with clinical chorioamnionitis, 95 to mothers with histological chorioamnionitis, and 146 to mothers with no chorioamnionitis. Data were available for 180 (78\%) of the 230 survivors at 18 months corrected age. Infants in the study groups were similar in gestational age, birth weight, and sex distribution. Clinical and histological chorioamnionitis were not predictive of infant mortality, cerebral palsy, bronchopulmonary dysplasia, periventricular leukomalacia, or retinopathy of prematurity. Infants in the clinical chorioamnionitis group had significantly lower cognitive ( $88 \pm 10)$, language $(82 \pm 12)$, and motor $(89 \pm 11)$ scores compared with infants in the histological chorioamnionitis group $(101 \pm 13, p<0.01$; $91 \pm 13, p<0.05$; and $99 \pm 13, p<0.05$, respectively) and to infants in the no chorioamnionitis group (99 $\pm 13, p<0.01 ; 92 \pm 15, p<0.05$; and $97 \pm 13, p<0.05$, respectively). Clinical chorioamnionitis is associated with developmental delay in preterm infants despite adequate treatment.
\end{abstract}

Chorioamnionitis is a major cause of maternal and neonatal morbidity. The term refers to inflammation of the amniotic fluid, membranes, placenta, and/or decidua and has been strongly associated with premature labor, occurring in $30 \%$ of babies born after premature labor with intact membranes and in $75 \%$ with premature rupture of membranes (PROM). ${ }^{1}$ The incidence of chorioamnionitis varies considerably de- pending on the gestational age at the time of delivery, with rates as high as $41 \%$ in those born at less than 27 weeks' gestation, $15 \%$ between 28 to 36 weeks' gestation, and $2 \%$ at term gestation. ${ }^{2}$ Most cases of chorioamnionitis are a consequence of intra-amniotic infection that has four main sources including ascending infection through the vagina, hematogenous spread through the placenta, retrograde seeding from received

December 21, 2011

accepted after revision

March 20, 2012

published online

July 6, 2012
Copyright $\odot 2013$ by Thieme Medical Publishers, Inc., 333 Seventh Avenue, New York, NY 10001, USA. Tel: +1(212) 584-4662.
DOI http://dx.doi.org/ 10.1055/s-0032-1321501. ISSN $0735-1631$. 
the peritoneal cavity through the fallopian tubes, and accidental introduction of pathogens during an invasive procedure. $^{3}$

Chorioamnionitis has been shown to be a risk factor for various morbidities including neonatal sepsis, ${ }^{4}$ respiratory distress syndrome, ${ }^{5}$ bronchopulmonary dysplasia (BPD), ${ }^{6}$ neonatal meningitis, ${ }^{7}$ intraventricular hemorrhage (IVH), ${ }^{8}$ periventricular leukomalacia (PVL), ${ }^{9}$ cerebral palsy (CP), ${ }^{10}$ retinopathy of prematurity (ROP), ${ }^{11}$ and developmental delay. ${ }^{12-14}$ Short- and long-term sequelae of chorioamnionitis vary considerably with gestational age. Full-term infants exposed to chorioamnionitis are at an increased risk of perinatal depression, encephalopathy, and $\mathrm{CP} .{ }^{15-17}$ On the other hand, preterm infants are at an increased risk of IVH and PVL, which can ultimately lead to mental retardation and $\mathrm{CP}{ }^{18,19}$ Suggested theories to explain the association between maternal chorioamnionitis and neonatal morbidities include the primary endotoxic effect of the invading pathogens, placental abruption, fetal vascular inflammation, fetal cardiac dysfunction, increased fetal oxygen consumption related to maternal fever, and fetal inflammatory response syndrome. ${ }^{20-24}$

Various measures have been made to screen for and prevent intra-amniotic infection during pregnancy aiming to minimize chorioamnionitis and associated maternal and neonatal morbidities. These measures include screening and management of urinary tract infection throughout the pregnancy, ${ }^{25,26}$ the use of antibiotics in patients with PROM who are being managed expectantly, ${ }^{27,28}$ development of and adherence to chemoprophylaxis guidelines for group B streptococcal (GBS) colonization, ${ }^{29,30}$ and intrapartum antibiotics for clinical chorioamnionitis. ${ }^{31,32}$ The efficacy of these measures in reducing maternal and neonatal morbidities shows considerable variation among different trials. Administration of antibiotics in patients with PROM was associated with a significant reduction in the incidence of histological chorioamnionitis but did not modify the frequency of funisitis. ${ }^{33}$ Treatment of bacteriuria in pregnant women was found to clear the asymptomatic bacteriuria, lower the incidence of maternal pyelonephritis, and reduce the incidence of low-birth-weight infants. ${ }^{34}$ Antibiotics prescribed for women with PROM were associated with some short-term benefits like prolongation of pregnancy, less infection, and less abnormal cerebral ultrasound before discharge from hospital. ${ }^{35,36}$ Intrapartum antibiotic treatment of women colonized with GBS decreased the rate of infant colonization and early onset neonatal infection with GBS. ${ }^{37}$ Administration of broadspectrum antibiotics for clinical chorioamnionitis has been associated with significant reduction in neonatal sepsis and pneumonia. $^{38}$

We hypothesized that early detection and strict management of intra-amniotic infection would improve the outcome of preterm infants. Accordingly, our objective was to evaluate if prepartum prophylaxis against intra-amniotic infection and intrapartum management of chorioamnionitis improves the mortality and morbidity of preterm infants.

\section{Subjects and Methods}

\section{Study Design}

This was a retrospective cohort study aiming to compare the outcomes of preterm infants born to mothers with versus those born to mothers without chorioamnionitis at Sunnybrook Health Sciences Centre in Toronto, Canada. Charts for infants and mothers were reviewed for the accuracy of maternal and neonatal data and the presence of developmental assessment at 18 months' corrected gestational age. The study was approved by the Research Ethics Boards at Sunnybrook Health Sciences Centre.

\section{Subjects and Data}

Preterm infants born at less than 30 weeks' gestation who were admitted to the Neonatal Intensive Care Unit (NICU) of Sunnybrook Health Sciences Centre between January 2007 and December 2008 were included. No formal power analysis or sample size estimate was performed.

A study protocol was designed in which maternal and neonatal data were retrospectively collected. Maternal data included: presence and duration of fever $\left(>38^{\circ} \mathrm{C}\right)$ prior to delivery, last recorded white blood cell count, evidence for urinary tract infection (proven by positive urine culture) at any time during pregnancy, maternal GBS status, presence and duration of rupture of membranes, foul odor of the amniotic fluid, obstetric diagnosis of clinical chorioamnionitis, pre- and intrapartum antibiotics prescribed for any intraamniotic infection, mode of delivery, treatment with antenatal steroids, and placental pathology as determined by a perinatal pathologist. Neonatal data included: gestational age, sex, weight at birth, birth weight in relation to gestational age, and Apgar score at 1 and 5 minutes. Neonatal outcomes included mortality (defined as death before hospital discharge), CP, early onset sepsis, BPD (defined as oxygen requirement at 36 weeks' corrected gestational age), ${ }^{39}$ prethreshold or threshold $\mathrm{ROP}^{40}$ unilateral or bilateral hearing impairment, any grade IVH and ventricular dilatation as detected by head ultrasound, ${ }^{41}$ PVL by head ultrasound, ${ }^{42}$ and neurodevelopmental assessment at 18 months' corrected age assessed by the Bayley Scales of Infant Development (third edition) (BSID-III). ${ }^{43}$

Depending on presence or absence of chorioamnionitis, preterm infants were divided into three groups: clinical chorioamnionitis, histological chorioamnionitis, or no chorioamnionitis. Clinical chorioamnionitis was defined as the presence of maternal fever of greater than $38^{\circ} \mathrm{C}\left(100.4^{\circ} \mathrm{F}\right)$ and two of the following conditions: maternal leukocytosis (greater than 15,000 cells $/ \mathrm{mm}^{3}$ ), maternal tachycardia (greater than 100 beats/min), fetal tachycardia (greater than 160 beats/min), uterine tenderness, and foul odor of the amniotic fluid. ${ }^{2}$ Histological chorioamnionitis was defined as the presence of inflammation of the chorioamnion or the umbilical cord (funisitis), chorionic vasculitis, and umbilical phlebitis or vasculitis in the placental pathology. ${ }^{44}$ Women with both clinical and histological chorioamnionitis were included in the clinical chorioamnionitis group. 


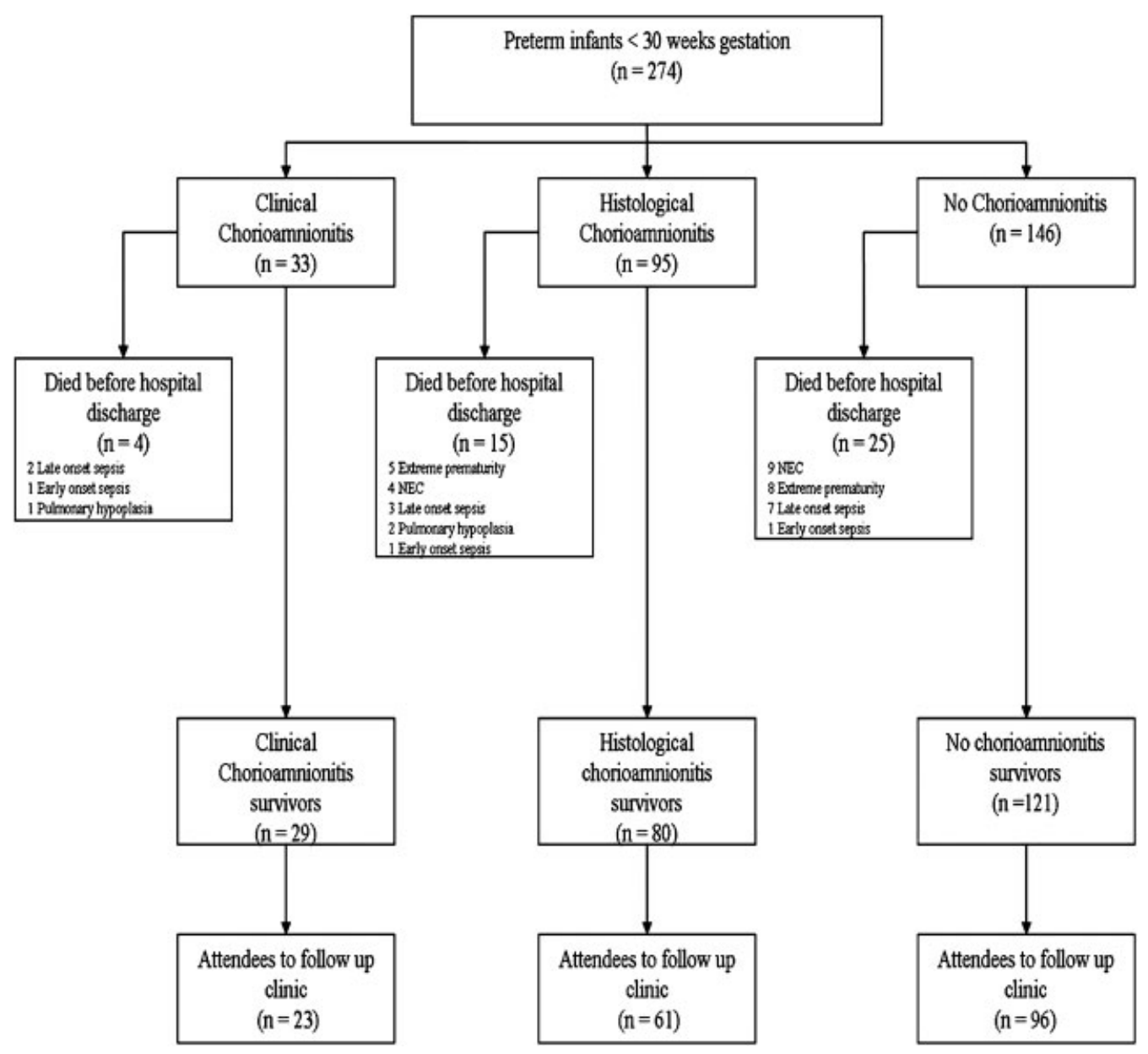

Figure 1 Study group characteristics. NEC, necrotizing enterocolitis.

\section{Statistical Analysis}

Differences between the study groups were assessed using the analysis of variance with Tukey test for continuous variables, and the chi-square $\left(\mathrm{x}^{2}\right)$ test with Fisher exact for categorical variables. Odds ratios (ORs) and 95\% confidence intervals (CIs) were calculated to assess the magnitude of the differences. Spearman test was used to assess the correlation between developmental outcome and risk factors. KaplanMeier survival analysis was used to compare probability of survival between studied groups over time. Unless otherwise stated, values are expressed as means \pm standard deviations or absolute numbers and percentages. Analysis was performed using SPSS statistical software (version 16; SPSS, Chicago, IL). We did not do a formal power analysis or sample size estimation. $p$ values of $<0.05$ were considered statistically significant.

\section{Results}

We identified 274 preterm infants with a gestational age of less than 30 weeks (mean $27 \pm 1.6$ weeks and a mean birth weight of $952 \pm 269 \mathrm{~g}$ ) during the study period. Out of this cohort, 33 (12\%) infants were born to mothers with clinical chorioamnionitis, 95 (35\%) infants were born to mothers with histological chorioamnionitis, and 146 (53\%) infants were born to mothers with no chorioamnionitis. Of these infants, 230 (84\%) survived to hospital discharge. Data were available for 180 (78\% of survivors) at 18 months' corrected age who attended the follow-up clinic. Of those survivors, 23 were in the clinical chorioamnionitis group, 61 in the histological group, and 96 in the no chorioamnionitis group as shown in - Fig. 1.

Prolonged PROM was significantly higher in the clinical $(n=21,64 \%, p<0.001)$ and histological $(n=45,47 \%$, $p<0.001$ ) chorioamnionitis groups compared with the no chorioamnionitis $(n=25,17 \%)$ group (-Table 1). Urinary tract infection was not significantly different between the studied groups representing 4 (12\%), 14 (15\%), and 9 (6\%) mothers in the clinical, histological, and no chorioamnionitis groups respectively. GBS screening was positive in 5 (15\%), 16 (17\%), and 20 (14\%) mothers with clinical, histological, and no chorioamnionitis, respectively.

Prepartum antibiotics were prescribed to 28 (85\%) mothers with clinical and to 70 (75\%) mothers with histological chorioamnionitis (-Table 1). Most of them were given for PROM. All mothers with clinical chorioamnionitis were treated with intrapartum antibiotics.

Infants born to mothers with clinical and histological chorioamnionitis had similar gestational ages, birth weights, 
Table 1 Baseline Characteristics of Preterm Infants

\begin{tabular}{|c|c|c|c|}
\hline Characteristics & $\begin{array}{l}\text { Clinical Chorioamnionitis } \\
(n=33)\end{array}$ & $\begin{array}{l}\text { Histological Chorioamnionitis } \\
(n=95)\end{array}$ & $\begin{array}{l}\text { No Chorioamnionitis } \\
(n=146)\end{array}$ \\
\hline Gestational age (wk) & $27.3 \pm 1.3$ & $27.0 \pm 1.7$ & $27.1 \pm 1.7$ \\
\hline Birth weight (g) & $988 \pm 226$ & $976 \pm 273$ & $927 \pm 275$ \\
\hline Male & $15(46 \%)$ & $54(56 \%)$ & $83(57 \%)$ \\
\hline PROM & $21(64 \%)^{\mathrm{a}}$ & $45(47 \%)^{\mathrm{a}}$ & $25(17 \%)$ \\
\hline \multicolumn{4}{|l|}{ Apgar score } \\
\hline $1 \mathrm{~min}$ & $5.2 \pm 2.4$ & $5.5 \pm 2.4$ & $5.6 \pm 2.3$ \\
\hline $5 \mathrm{~min}$ & $6.8 \pm 2.0$ & $7.2 \pm 1.8$ & $7.5 \pm 1.7$ \\
\hline \multicolumn{4}{|l|}{ Antenatal steroid } \\
\hline Two doses & 18 (55\%) & $58(61 \%)$ & $103(71 \%)$ \\
\hline One dose & $7(21 \%)$ & $19(20 \%)$ & $27(18 \%)$ \\
\hline None & $8(24 \%)$ & $18(19 \%)$ & $16(11 \%)$ \\
\hline \multicolumn{4}{|l|}{ Mode of delivery } \\
\hline VD & $14(42 \%)^{a}$ & $51(54 \%)^{a}$ & 27 (19\%) \\
\hline Forceps & $0(0 \%)$ & $2(2 \%)$ & $0(0 \%)$ \\
\hline Vacuum & $0(0 \%)$ & $0(0 \%)$ & $1(0.5 \%)$ \\
\hline CS (no PTL) & $0(0 \%)^{a}$ & $5(5 \%)^{a}$ & $59(40 \%)$ \\
\hline CS (PTL) & $19(58 \%)$ & $37(37 \%)$ & $59(40 \%)$ \\
\hline \multicolumn{4}{|l|}{ Weight for gestation } \\
\hline SGA & $5(15 \%)$ & $8(9 \%)^{a}$ & $39(27 \%)$ \\
\hline AGA & $28(85 \%)$ & $86(91 \%)^{a}$ & $104(71 \%)$ \\
\hline LGA & $0(0 \%)$ & $0(0 \%)$ & $3(2 \%)$ \\
\hline \multicolumn{4}{|l|}{ Maternal antibiotics therapy } \\
\hline Prepartum prophylactic & $28(85 \%)^{\mathrm{a}}$ & $70(74 \%)^{\mathrm{a}}$ & $27(18)$ \\
\hline Intrapartum therapeutic & $33(100 \%)^{a}$ & $0(0 \%)$ & $0(0 \%)$ \\
\hline
\end{tabular}

Data expressed as mean \pm standard deviation or $n$ (\%). PROM, premature rupture of membranes; VD, vaginal delivery; CS, cesarean section; PTL, preterm labor; SGA, small for gestational age; AGA, average for gestational age; LGA, large for gestational age.

${ }^{a} p<0.05$ by analysis of variance or chi-square test compared with no chorioamnionitis group.

sex distribution, exposure to antenatal steroid therapy, and Apgar scores compared with infants in the no chorioamnionitis groups ( Table1). Premature labor resulting in vaginal delivery was significantly associated with clinical $(n=14$, $42 \%, p<0.00)$ and histological chorioamnionitis $(n=51$, $54 \%, p<0.00)$ groups compared with no chorioamnionitis ( $n=27,19 \%$ ) group (-Table 1).

There were no differences in mortality rates between the three groups (-Table $\mathbf{2}$ ). Kaplan-Meier estimates of cumulative mortality produced similar results ( - Fig. 2 ) indicating no overall effect of chorioamnionitis on incidence or timing of death.

Extreme prematurity and necrotizing enterocolitis were the main causes of mortality during NICU admission among the full cohort ( $\mathbf{- F i g . 1} \mathbf{1}$ ).

When adjusted for mode of delivery and PROM, intraventricular hemorrhage (particularly grades 1 and 2) was significantly higher in the clinical ( $n=13,39 \%)$ and histological ( $n=26,27 \%$ ) chorioamnionitis groups compared with no chorioamnionitis $(n=23,16 \%)$ group (-Table 2 ); other short-term neonatal outcomes including BPD, ROP, and early onset sepsis were not different between the studied groups (-Table 2). Also, long-term outcomes including CP and hearing impairment were not statistically different between the studied groups (-Table $\mathbf{3}$ ).

Neurodevelopmental assessment by BSID-III at 18 months' corrected age revealed a significantly higher number of infants whose cognitive, language, and motor scores were below average in the clinical chorioamnionitis group when compared with infants in the no chorioamnionitis groups (-Table 3). There was a significantly higher number of infants whose language scores were severely impaired (below a composite score of 70) in the clinical chorioamnionitis group when compared with infants in the no chorioamnionitis groups (-Table $\mathbf{3}$ ). Infants in the clinical chorioamnionitis group had significantly lower cognitive $(88 \pm 10)$, language ( $82 \pm 12$ ), and motor $(89 \pm 11)$ scores compared with infants in the histological chorioamnionitis group (101 \pm 13 , $p<0.01 ; 91 \pm 13, p<0.05$; and $99 \pm 13, p<0.05$, respectively) and to infants in the no chorioamnionitis group 
Table 2 Impact of Chorioamnionitis on Short-Term Outcomes of Preterm Infants

\begin{tabular}{|c|c|c|c|c|c|c|c|c|c|}
\hline \multirow[t]{2}{*}{ Variable } & \multicolumn{4}{|c|}{$\begin{array}{l}\text { Clinical Chorioamnionitis } \\
\qquad(n=33)\end{array}$} & \multicolumn{4}{|c|}{$\begin{array}{l}\text { Histological Chorioamnionitis } \\
\qquad(n=95)\end{array}$} & \multirow{2}{*}{$\begin{array}{l}\text { No Chorioamnionitis } \\
(n=146) \\
n(\%)\end{array}$} \\
\hline & $n(\%)$ & OR & $95 \% \mathrm{Cl}$ & $p$ & $n(\%)$ & OR & $95 \% \mathrm{Cl}$ & $p$ & \\
\hline Mortality & $4(12 \%)$ & 0.6 & $0.1-2.1$ & 0.47 & $15(16 \%)$ & 0.8 & $0.3-1.7$ & 0.59 & $25(17 \%)$ \\
\hline Chronic lung disease & $4(12 \%)$ & 0.3 & $0.11-1.2$ & 0.11 & $21(22 \%)$ & 0.8 & $0.4-1.6$ & 0.57 & $38(26 \%)$ \\
\hline \multicolumn{10}{|c|}{ Retinopathy of prematurity } \\
\hline Prethreshold & $6(18 \%)$ & 1.3 & $0.4-4.0$ & 0.55 & 15 (16\%) & 1.7 & $0.8-3.7$ & 0.14 & $35(24 \%)$ \\
\hline Threshold & $1(3 \%)$ & 0.2 & $0.01-2.4$ & 0.20 & $1(1 \%)$ & 0.9 & $0.1-11.9$ & 0.94 & $2(1 \%)$ \\
\hline \multicolumn{10}{|c|}{ Head ultrasound results } \\
\hline Any grade IVH & $13(39 \%)$ & 0.3 & $0.1-0.7$ & $0.01^{\mathrm{a}}$ & $26(27 \%)$ & 0.4 & $0.2-0.94$ & $0.03^{a}$ & $23(16 \%)$ \\
\hline Grade 3-4 IVH & $0(0 \%)$ & 0 & 0 & 0 & $0(0 \%)$ & 0 & 0 & 0 & $3(2 \%)$ \\
\hline Ventriculomegaly & $3(9 \%)$ & 0.2 & $0.04-1.4$ & 0.18 & $0(0 \%)$ & 0 & 0 & 0 & $5(3 \%)$ \\
\hline PVL & $1(3 \%)$ & 0.3 & $0.01-10.5$ & 0.54 & $3(3 \%)$ & 0.3 & $0.02-3.3$ & 0.32 & $1(0.5 \%)$ \\
\hline Early onset sepsis & $3(9 \%)$ & 0.3 & $0.06-1.9$ & 0.24 & $4(4 \%)$ & 0.7 & $0.16-3.7$ & 0.7 & $5(3 \%$ \\
\hline
\end{tabular}

IVH, intraventricular hemorrhage; PVL, periventricular leukomalacia.

${ }^{a} p<0.05$ by multinomial logistic regression adjusted for mode of delivery and presence of premature rupture of membranes.

$(99 \pm 13, \quad p<0.01 ; \quad 92 \pm 15, \quad p<0.05 ; \quad$ and $97 \pm 13$, $p<0.05$, respectively; - Fig. 3 ).

When the results were reviewed after exclusion of cases with early onset sepsis, cognitive, language, and motor scores remained significantly lower in the clinical chorioamnionitis group compared with infants in the histological chorioamnionitis and the no chorioamnionitis groups. Cognitive, language, and motor scores were not different between infants in the histological chorioamnionitis and no chorioamnionitis groups.

Within the histological chorioamnionitis group, neurodevelopmental outcomes did not show any significant difference in correlation with stages of histological chorioamnionitis (i.e.,

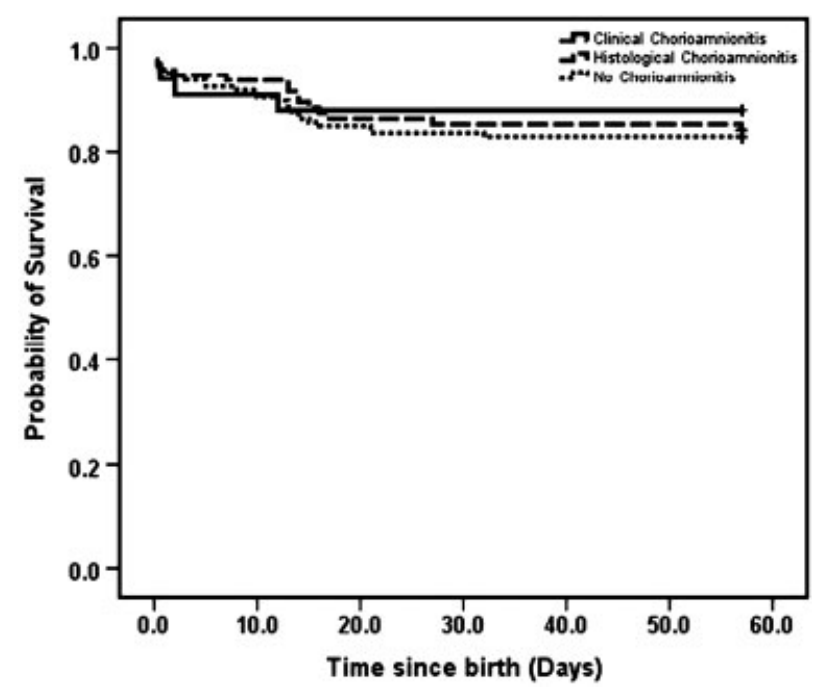

Figure 2 Kaplan-Meier survival curves for infants according to studied group. A log-rank test for homogeneity of the survival curves was conducted over the 60-day period $(p=0.8)$. chorionitis versus amnionitis or funisitis; - Table 4). Cognitive, language, and motor scores did not show any significant relation or association with the duration of rupture of amniotic membranes or duration of maternal fever at time of birth in the clinical or histological chorioamnionitis group.

\section{Discussion}

Prevention and management of intra-amniotic infection has gained considerable attention in the past few years. However, the efficacy of preventive and therapeutic measures in minimizing neonatal morbidities varies among previous reports. Our main finding is that despite adequate intrapartum treatment, clinical chorioamnionitis is associated with neurodevelopmental delay in preterm infants. This finding is consistent with previous reports of the association between maternal chorioamnionitis and poor neurodevelopmental outcome of preterm infants. ${ }^{45-47}$

Ascending infection, the most common route leading to chorioamnionitis, progresses through several stages starting with colonization of the vagina by pathogenic strains, invasion and proliferation in the decidua, inflammation of amniotic membranes (amnionitis), inflammation of the umbilical cord (funisitis), fetal vasculitis, and finally bacteria and/or their endotoxins gaining access to the fetus through the amniotic fluid or fetal vessels. ${ }^{3}$ Accordingly, it is reasonable to think that fetal involvement and outcome would be proportionate to the severity and stage of chorioamnionitis, with clinical chorioamnionitis being more severe than the histological stage. To assess this, we compared the outcome of preterm infants born to mothers with either clinical or histological chorioamnionitis to those with no chorioamnionitis.

The impact of histological chorioamnionitis on the outcome of preterm infants has been inconsistent among 
Table 3 Impact of Chorioamnionitis on Long-Term Outcomes of Preterm Infants

\begin{tabular}{|c|c|c|c|c|c|c|c|c|c|}
\hline \multirow[t]{2}{*}{ Variable } & \multicolumn{4}{|c|}{$\begin{array}{l}\text { Clinical Chorioamnionitis } \\
(n=33)\end{array}$} & \multicolumn{4}{|c|}{$\begin{array}{l}\text { Histological Chorioamnionitis } \\
(n=95)\end{array}$} & \multirow{2}{*}{$\begin{array}{l}\text { No Chorioamnionitis } \\
(n=146) \\
n(\%)\end{array}$} \\
\hline & $n(\%)$ & OR & $95 \% \mathrm{Cl}$ & $p$ & $n(\%)$ & OR & $95 \% \mathrm{Cl}$ & $p$ & \\
\hline Cerebral palsy & $2(6 \%)$ & 1.3 & $0.2-7.9$ & 0.72 & $2(2 \%)$ & 0.4 & $0.08-2.1$ & 0.3 & $9(6 \%)$ \\
\hline \multicolumn{10}{|c|}{ Hearing impairment } \\
\hline Unilateral & $2(6 \%)$ & 0.1 & $0.01-1.1$ & 0.06 & $0(0 \%)$ & 0 & 0 & 0 & $2(1 \%)$ \\
\hline Bilateral & $0(0 \%)$ & 0 & 0 & 0 & $3(3 \%)$ & 2.2 & $0.4-9.8$ & 0.29 & $7(5 \%)$ \\
\hline \multicolumn{10}{|c|}{ BSID-III score below average for age } \\
\hline Cognitive & $9(27 \%)$ & 0.2 & $0.06-0.9$ & $0.04^{\mathrm{a}}$ & $6(6 \%)$ & 2.1 & $0.6-7.1$ & 0.2 & $14(9 \%)$ \\
\hline Language & $15(45 \%)$ & 0.3 & $0.09-1.0$ & $0.04^{\mathrm{a}}$ & $18(19 \%)$ & 1.2 & $0.5-2.6$ & 0.6 & $21(14 \%)$ \\
\hline Motor & $6(18 \%)$ & 0.2 & $0.04-0.8$ & $0.03^{\mathrm{a}}$ & $6(6 \%)$ & 1 & $0.3-3.5$ & 0.9 & $11(7 \%)$ \\
\hline \multicolumn{10}{|c|}{ BSID-III score below 70} \\
\hline Cognitive & $0(0 \%)$ & 0 & 0 & 0 & $0(0 \%)$ & 0 & 0 & 0 & $3(2 \%)$ \\
\hline Language & $4(12 \%)$ & 0.1 & $0.02-0.7$ & $0.02^{\mathrm{a}}$ & $4(4 \%)$ & 0.9 & $0.2-5.0$ & 0.9 & $5(3 \%)$ \\
\hline Motor & $1(3 \%)$ & 0.8 & $0.6-12.2$ & 0.9 & $1(1 \%)$ & 1.6 & $0.1-16.9$ & 0.6 & $4(3 \%)$ \\
\hline
\end{tabular}

BSID-III, Bayley Scales of Infant Development third edition; Cl, confidence interval; OR, odds ratio.

${ }^{a} p<0.05$ compared with no chorioamnionitis group by multinomial logistic regression adjusted for mode of delivery and presence of premature rupture of membranes.

previous research reports. We found that histological chorioamnionitis was not associated with increases in any of the neonatal morbidities or impaired neurodevelopmental outcomes in our cohort of infants. Previous reports have shown that histological chorioamnionitis was associated with higher incidences of IVH and ROP in preterm infants but had no effect on mental and psychomotor developmental indices or risk of $\mathrm{CP}^{14,19}$ Other reports revealed that histological chorioamnionitis was not associated with increase in the risk of IVH. ${ }^{48}$ The inconsistency among previous reports may be related to the broad definition used for histological chorioamnionitis

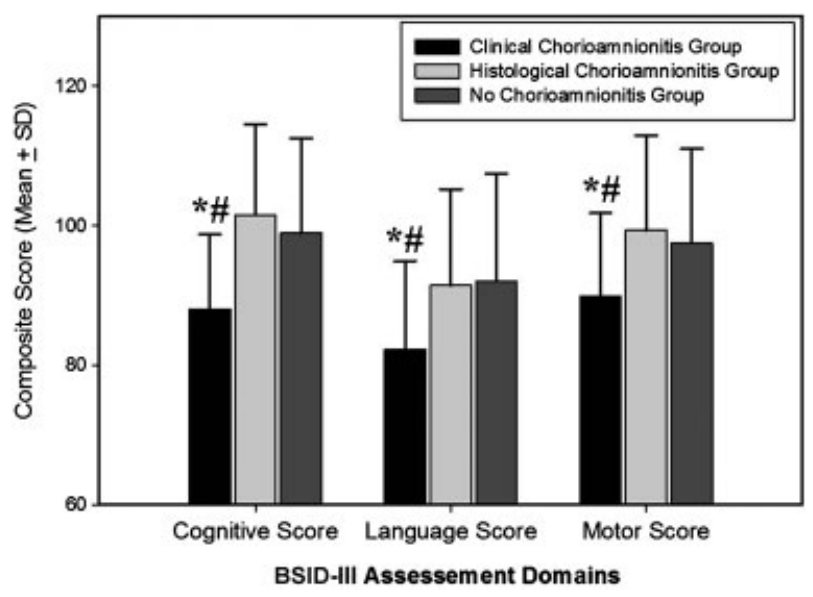

Figure 3 Mean Bayley Scales of Infant Development (third edition) (BSID-III) composite score for studied preterm infants at 18 months' corrected age. SD, standard deviation. ( ${ }^{*} p<0.05$ by analysis of variance with Tukey test compared with no chorioamnionitis group; ${ }^{\#} p<0.05$ by analysis of variance with Tukey test compared with histological chorioamnionitis group.) rather than to the stage. Rovira et al found that funisitis carried a higher risk of moderate to severe disability at 2 years of age in preterm infants compared with other stages of histological chorioamnionitis. ${ }^{45}$ Pacora et al found that newborns whose umbilical cords have evidence of funisitis had higher rates of clinical chorioamnionitis and neonatal sepsis than in those who did not ( $40 \%$ versus $8 \%$, and $40 \%$ versus $4 \%$, respectively; $p<0.01) .{ }^{49}$ Park et al found that the fetal and intra-amniotic inflammatory response was more intense with the involvement of the amnion rather than chorionitis alone. ${ }^{50}$ Accordingly, fetal infection and consequent adverse neonatal outcome were more highly correlated with invasion and inflammation closer to the fetus. In contrast to the previous reports, we did not find any difference in neonatal outcomes between different stages of histological chorioamnionitis in our cohort.

Unlike histological chorioamnionitis, clinical chorioamnionitis has a more consistent deleterious effect on neonatal outcome. $^{18,51}$ In a meta-analysis of $\sim 30$ trials, clinical chorioamnionitis was associated with increased risk of PVL (relative risk [RR], 3.0; 95\% CI, 2.2 to 4.0 ) and CP in babies born preterm (RR, 1.9; 95\% CI, 1.4 to 2.5 ) and at term (RR, 4.7; 95\% CI, 1.3 to 16.2$){ }^{9}$ In comparison to previous reports, clinical chorioamnionitis did not increase the risk of PVL, $\mathrm{CP}$, or any of the major neonatal morbidities in our cohort. However, despite adequate treatment, infants who were born to mothers with clinical chorioamnionitis had lower cognitive, language, and motor development scores compared with those without clinical chorioamnionitis.

Although invading pathogens and their endotoxic products are suggested as a possible cause for fetal injury in chorioamnionitis, ${ }^{20}$ the fetal inflammatory response has currently been receiving greater consideration as a cause of 
Table 4 Outcome of Preterm Infants among Various Stages of Histological Chorioamnionitis

\begin{tabular}{|l|l|l|l|}
\hline BSID-III & Chorionitis $(\boldsymbol{n}=\mathbf{4 0})$ & Amnionitis $(\boldsymbol{n}=10)$ & Funisitis $(\boldsymbol{n}=\mathbf{4 5})$ \\
\hline Cognitive score & $100.0 \pm 13.1$ & $97.0 \pm 14.0$ & $98.0 \pm 13.9$ \\
\hline Language score & $92.7 \pm 14.5$ & $88.3 \pm 14.1$ & $90.4 \pm 13.6$ \\
\hline Motor score & $97.1 \pm 19.1$ & $96.7 \pm 14.7$ & $98.4 \pm 14.1$ \\
\hline
\end{tabular}

Data expressed as mean \pm standard deviation or $n(\%)$. There were no statistically significant differences between groups. BSID-III, Bayley Scales of Infant Development, third edition.

fetal injury. ${ }^{3}$ As a result of this inflammatory response, cytokines (interleukins- 1 and -6 ; tumor necrosis factor- $\alpha$ ) are increased in the fetal blood, potentially initiating necrosis of white matter in the fetal brain and leading to long-term neurological sequelae, including $\mathrm{CP}^{52}$ Gomez et al demonstrated that the severity of the fetal inflammatory response syndrome, presented by the level of fetal plasma interleukin$6(>11 \mathrm{pg} / \mathrm{mg})$, was an independent predictor of severe neonatal morbidity (OR, 4.3; $95 \% \mathrm{CI}, 1.0$ to 18.5 ) and of PTL. ${ }^{53}$ Our finding of poor neurodevelopmental outcomes in the clinical chorioamnionitis group despite adequate treatment and the lack of association between neurodevelopmental outcomes and the subgroups with histological chorioamnionitis support the previous reports that fetal inflammatory response syndrome is more intense with clinical chorioamnionitis and is more predicative of poor outcome in the preterm infant. Accordingly, adequate peripartum treatment of chorioamnionitis may suppress the fetal inflammatory response syndrome, decrease the levels of inflammatory cytokines, and minimize neonatal sequelae. A proper study design to prove this theory will be difficult, as it will be unethical to have a control group of inadequately treated pregnant mothers to compare the level of fetal inflammatory response and neonatal outcome to an adequately treated group. However, previous reports have shown that antenatal measures that decrease the fetal inflammatory response syndrome are associated with better neonatal outcomes. Antenatal corticosteroids administered in preterm birth associated with chorioamnionitis have been shown to decrease the incidence of severe IVH (OR, $0.29 ; 95 \% \mathrm{CI}, 0.10$ to 0.89 ; $p=0.03$ ) and periventricular leukomalacia (OR, $0.35 ; 95 \% \mathrm{CI}$, 0.14 to $0.85 ; p=0.02)^{54}$

Our results are limited by a moderate loss to follow-up (22\%), which may justify repeated analysis with and without missing data. Because the percentage of morbidity seen in babies who attend versus those who do not attend follow-up varies between reports and is unknown in our cohort; we did not want to make any assumptions about the incidence of the various outcomes in those infants seen versus those who failed to attend. Another limitation is the retrospective design of the study, which allows for personal bias in the decision to administer pre- or intrapartum antibiotics. However, this bias is limited by a clear hospital policy in defining chorioamnionitis and close adherence to an approved management protocol.

In conclusion, clinical chorioamnionitis is associated with poor neurodevelopmental outcome in preterm infants de- spite adequate treatment. Future research is needed to explore sensitive and specific markers for the initiation of fetal inflammatory response syndrome and the correlation with neonatal outcome. Detection of early markers of impending clinical chorioamnionitis may allow for preemptive treatment with resultant improvement in neonatal outcome.

\section{Authors' Contributions}

Nehad Nasef participated in formulating the hypothesis, design of the study, data collection, data interpretation, statistical analysis, and writing of the manuscript. Abd Elazeez Shabaan, Patti Schurr, Dolores Iaboni, and Julie Choudhury participated in design of the study, data collection, and writing the manuscript. Paige Church and Michael Dunn participated in study design, data interpretation, and writing of the manuscript. All authors have read and approved the final manuscript.

\section{References}

1 Yoon BH, Romero R, Moon JB, et al. Clinical significance of intraamniotic inflammation in patients with preterm labor and intact membranes. Am J Obstet Gynecol 2001;185:1130-1136

2 Newton ER. Chorioamnionitis and intraamniotic infection. Clin Obstet Gynecol 1993;36:795-808

3 Arad I, Ergaz Z. The fetal inflammatory response syndrome and associated infant morbidity. Isr Med Assoc J 2004;6:766-769

4 Yoder PR, Gibbs RS, Blanco JD, Castaneda YS, St Clair PJ. A prospective, controlled study of maternal and perinatal outcome after intra-amniotic infection at term. Am J Obstet Gynecol 1983;145:695-701

5 Yoon BH, Romero R, Kim KS, et al. A systemic fetal inflammatory response and the development of bronchopulmonary dysplasia. Am J Obstet Gynecol 1999;181:773-779

6 Jobe AH. Antenatal associations with lung maturation and infection. J Perinatol 2005;25(Suppl 2):S31-S35

7 Aziz N, Cheng YW, Caughey AB. Neonatal outcomes in the setting of preterm premature rupture of membranes complicated by chorioamnionitis. J Matern Fetal Neonatal Med 2009;22: 780-784

8 Moscuzza F, Belcari F, Nardini V, et al. Correlation between placental histopathology and fetal/neonatal outcome: chorioamnionitis and funisitis are associated to intraventricular haemorrage and retinopathy of prematurity in preterm newborns. Gynecol Endocrinol 2011;27:319-323

9 Wu YW, Colford JM Jr. Chorioamnionitis as a risk factor for cerebral palsy: a meta-analysis. JAMA 2000;284:1417-1424

10 Shatrov JG, Birch SC, Lam LT, Quinlivan JA, McIntyre S, Mendz GL. Chorioamnionitis and cerebral palsy: a meta-analysis. Obstet Gynecol 2010;116(2 Pt 1):387-392 
11 Moscuzza F, Belcari F, Nardini V, et al. Correlation between placental histopathology and fetal/neonatal outcome: chorioamnionitis and funisitis are associated to intraventricular haemorrage and retinopathy of prematurity in preterm newborns. Gynecol Endocrinol 2011;27:319-323

12 Adams-Chapman I, Stoll BJ. Neonatal infection and long-term neurodevelopmental outcome in the preterm infant. Curr Opin Infect Dis 2006;19:290-297

13 Fung G, Bawden K, Chow P, Yu V. Chorioamnionitis and outcome in extremely preterm infants. Ann Acad Med Singapore 2003;32: 305-310

$14 \mathrm{Mu}$ SC, Lin CH, Sung TC, et al. Neurodevelopmental outcome of very-low-birth-weight infants with chorioamnionitis. Acta Paediatr Taiwan 2007;48:207-212

15 Shalak LF, Perlman JM, Jackson GL, Laptook AR. Depression at birth in term infants exposed to maternal chorioamnionitis: does neonatal fever play a role? J Perinatol 2005;25:447-452

16 Shalak L, Johnson-Welch S, Perlman JM. Chorioamnionitis and neonatal encephalopathy in term infants with fetal acidemia: histopathologic correlations. Pediatr Neurol 2005;33:162-165

17 Wu YW, Escobar GJ, Grether JK, Croen LA, Greene JD, Newman TB. Chorioamnionitis and cerebral palsy in term and near-term infants. JAMA 2003;290:2677-2684

18 Soraisham AS, Singhal N, McMillan DD, Sauve RS, Lee SK; Canadian Neonatal Network. A multicenter study on the clinical outcome of chorioamnionitis in preterm infants. Am J Obstet Gynecol 2009; 200:372, e1-e6

19 Polam S, Koons A, Anwar M, Shen-Schwarz S, Hegyi T. Effect of chorioamnionitis on neurodevelopmental outcome in preterm infants. Arch Pediatr Adolesc Med 2005;159:1032-1035

20 Ilagan NB, Elias EG, Liang KC, Kazzi G, Piligian J, Khatib G. Perinatal and neonatal significance of bacteria-related placental villous edema. Acta Obstet Gynecol Scand 1990;69:287-290

21 Romero R, Espinoza J, Gonçalves LF, et al. Fetal cardiac dysfunction in preterm premature rupture of membranes. J Matern Fetal Neonatal Med 2004;16:146-157

22 Di Naro E, Cromi A, Ghezzi F, Giocolano A, Caringella A, Loverro G Myocardial dysfunction in fetuses exposed to intraamniotic infection: new insights from tissue Doppler and strain imaging. Am J Obstet Gynecol 2010;203:459, e1-e7

23 Rana A, Sawhney H, Gopalan S, Panigrahi D, Nijhawan R. Abruptio placentae and chorioamnionitis-microbiological and histologic correlation. Acta Obstet Gynecol Scand 1999;78:363-366

24 Darby MJ, Caritis SN, Shen-Schwarz S. Placental abruption in the preterm gestation: an association with chorioamnionitis. Obstet Gynecol 1989;74:88-92

25 Krcmery S, Hromec J, Demesova D. Treatment of lower urinary tract infection in pregnancy. Int J Antimicrob Agents 2001;17: 279-282

26 Vercaigne LM, Zhanel GG. Recommended treatment for urinary tract infection in pregnancy. Ann Pharmacother 1994;28:248-251

27 Mercer BM, Miodovnik M, Thurnau GR, et al; National Institute of Child Health and Human Development Maternal-Fetal Medicine Units Network. Antibiotic therapy for reduction of infant morbidity after preterm premature rupture of the membranes. A randomized controlled trial. JAMA 1997;278:989-995

28 Kenyon SL, Taylor DJ, Tarnow-Mordi W; ORACLE Collaborative Group. Broad-spectrum antibiotics for preterm, prelabour rupture of fetal membranes: the ORACLE I randomised trial. Lancet 2001; 357:979-988

29 American Academy of Pediatrics Committee on Infectious Diseases and Committee on Fetus and Newborn. Guidelines for prevention of group B streptococcal (GBS) infection by chemoprophylaxis. Pediatrics 1992;90:775-778

30 Gotoff SP, Boyer K. Combined, selective chemoprophylaxis of early onset neonatal group B streptococcal disease (GBS EOD). Adv Exp Med Biol 1997;418:267-268
31 Lyell DJ, Pullen K, Fuh K, et al. Daily compared with 8-hour gentamicin for the treatment of intrapartum chorioamnionitis: a randomized controlled trial. Obstet Gynecol 2010;115(2 Pt 1): 344-349

32 Fahey JO. Clinical management of intra-amniotic infection and chorioamnionitis: a review of the literature. J Midwifery Womens Health 2008;53:227-235

33 Ovalle A, Martínez MA, Kakarieka E, et al. Antibiotic administration in patients with preterm premature rupture of membranes reduces the rate of histological chorioamnionitis: a prospective, randomized, controlled study. J Matern Fetal Neonatal Med 2002; 12:35-41

34 Smaill F, Vazquez JC. Antibiotics for asymptomatic bacteriuria in pregnancy. Cochrane Database Syst Rev 2007;(2):CD000490

35 Kenyon S, Boulvain M, Neilson J. Antibiotics for preterm premature rupture of membranes. Cochrane Database Syst Rev 2001;(4): CD001058

36 Flenady V, King J. Antibiotics for prelabour rupture of membranes at or near term. Cochrane Database Syst Rev 2002;(3):CD001807

37 Smaill F. Intrapartum antibiotics for group B streptococcal colonisation. Cochrane Database Syst Rev 2000;(2):CD000115

38 Hopkins L, Smaill F. Antibiotic regimens for management of intraamniotic infection. Cochrane Database Syst Rev 2002;(3): CD003254

39 Jobe AH, Bancalari E. Bronchopulmonary dysplasia. Am J Respir Crit Care Med 2001;163:1723-1729

40 International Committee for the Classification of Retinopathy of Prematurity. The International Classification of Retinopathy of Prematurity revisited. Arch Ophthalmol 2005;123:991-999

41 Volpe JJ. Neurology of the Newborn. 5th ed. Philadelphia: Saunders/Elsevier; 2008

42 Veyrac C, Couture A, Saguintaah M, Baud C. Brain ultrasonography in the premature infant. Pediatr Radiol 2006;36:626-635

43 Macias MM, Saylor CF, Greer MK, Charles JM, Bell N, Katikaneni LD. Infant screening: the usefulness of the Bayley Infant Neurodevelopmental Screener and the Clinical Adaptive Test/Clinical Linguistic Auditory Milestone Scale. J Dev Behav Pediatr 1998;19: 155-161

44 Redline RW, Faye-Petersen O, Heller D, Qureshi F, Savell V, Vogler C; Society for Pediatric Pathology, Perinatal Section, Amniotic Fluid Infection Nosology Committee. Amniotic infection syndrome: nosology and reproducibility of placental reaction patterns. Pediatr Dev Pathol 2003;6:435-448

45 Rovira N, Alarcon A, Iriondo M, et al. Impact of histological chorioamnionitis, funisitis and clinical chorioamnionitis on neurodevelopmental outcome of preterm infants. Early Hum Dev 2011;87:253-257

46 Suppiej A, Franzoi M, Vedovato S, Marucco A, Chiarelli S, Zanardo V. Neurodevelopmental outcome in preterm histological chorioamnionitis. Early Hum Dev 2009;85:187-189

47 Kent A, Lomas F, Hurrion E, Dahlstrom JE. Antenatal steroids may reduce adverse neurological outcome following chorioamnionitis: neurodevelopmental outcome and chorioamnionitis in premature infants. J Paediatr Child Health 2005;41:186-190

48 Sarkar S, Kaplan C, Wiswell TE, Spitzer AR. Histological chorioamnionitis and the risk of early intraventricular hemorrhage in infants born $<$ or $=28$ weeks gestation. J Perinatol 2005;25: 749-752

49 Pacora P, Chaiworapongsa T, Maymon E, et al. Funisitis and chorionic vasculitis: the histological counterpart of the fetal inflammatory response syndrome. J Matern Fetal Neonatal Med 2002;11:18-25

50 Park CW, Moon KC, Park JS, Jun JK, Romero R, Yoon BH. The involvement of human amnion in histologic chorioamnionitis is an indicator that a fetal and an intra-amniotic inflammatory response is more likely and severe: clinical implications. Placenta 2009;30:56-61 
51 Botet F, Figueras J, Carbonell-Estrany X, Arca G; The Castrillo Study Group. Effect of maternal clinical chorioamnionitis on neonatal morbidity in very-low birthweight infants: a case-control study. J Perinat Med 2010;38:269-273

52 Shalak LF, Laptook AR, Jafri HS, Ramilo O, Perlman JM. Clinical chorioamnionitis, elevated cytokines, and brain injury in term infants. Pediatrics 2002;110:673-680
53 Gomez R, Romero R, Ghezzi F, Yoon BH, Mazor M, Berry SM. The fetal inflammatory response syndrome. Am J Obstet Gynecol 1998;179:194-202

54 Been JV, Degraeuwe PL, Kramer BW, Zimmermann LJ. Antenatal steroids and neonatal outcome after chorioamnionitis: a metaanalysis. BJOG 2011;118:113-122 